\title{
Diagnostic Accuracy of Fine Needle Aspiration Cytology versus Concurrent Core Needle Biopsy in Evaluation of Intrathoracic Lesions: a Retrospective Comparative Study
}

\author{
Arezoo Eftekhar-Javadi ${ }^{1}$,Perikala Vijayananda Kumar²,Ali Zare Mirzaie ${ }^{3}$,Amir \\ Radfar $^{4}$, Irina Filip ${ }^{5}$, Maximilian Niyazi ${ }^{6}$, Alireza Sadeghipour ${ }^{7 *}$
}

\begin{abstract}
Background: Transthoracic fine needle aspiration (FNA) cytology and core needle biopsy (CNB) are two commonly used approaches for the diagnosis of suspected neoplastic intrathoracic lesions. This study compared the diagnostic accuracy of FNA cytology and concurrent CNB in the evaluation of intrathoracic lesions. Materials and Methods: We studied FNA cytology and concurrent CNB specimens of 127 patients retrospectively, using hematoxylin and eosin (H\&E), immunohistochemistry, and, on certain occasions cytochemistry. Information regarding additional tissue tests was derived from the electronic archives of the Department of Pathology and Laboratory Medicine as well as patient records. Diagnostic accuracy was calculated for each test. Results: Of 127 cases, 22 were inconclusive and excluded from the study. The remaining 105 were categorized into 73 (69.5\%) malignant lesions and $32(30.5 \%)$ benign lesions. FNA and CNB findings were in complete agreement in 63 cases $(60 \%)$. The accuracy and confidence intervals (CIs) of FNA and CNB for malignant tumors were 86.3\% (CI: 79.3-90.7) and 93.2\% (CI: 87.3- 96.0 ) respectively. For epithelial malignant neoplasms, a definitive diagnosis was made in $44.8 \%$ of cases by FNA and $80.6 \%$ by CNB. The diagnostic accuracy of CNB for nonepithelial malignant neoplasms was $83.3 \%$ compared with $50 \%$ for FNA. Of the 32 benign cases, we made specific diagnoses in 16 with diagnostic accuracy of $81.3 \%$ and $6.3 \%$ for CNB and FNA, respectively. Conclusions: Our findings suggest that FNA is comparable to CNB in the diagnosis of malignant epithelial lesions whereas diagnostic accuracy of CNB for nonepithlial malignant neoplasms is superior to that for FNA. Further, for histological typing of tumors and examining tumor origin, immunohistochemical work up plays an important role.
\end{abstract}

Keywords: Fine needle aspiration - core needle biopsy - intrathoracic lesions - comparison

Asian Pac J Cancer Prev, 16 (16), 7385-7390

\section{Introduction}

Patients that are suspected of neoplastic lung disease require an accurate tissue diagnosis in order for an optimal treatment to be selected and applied. This diagnosis is usually prepared through percutaneous sampling. Improvements in radiological techniques have led to the identification of relatively small lesions, which are required as a guide to obtaining qualitative specimens. Transthoracic fine needle aspiration (FNA) cytology and core needle biopsy (CNB) are two commonly used methods for obtaining diagnostic material. FNA specimens are often acquired using 20-25 gauge needles, and these generally provide a sample for cytological examination, whereas CNB specimens are obtained using larger 14-18 gauge needles and they primarily provide a tissue core for histological assessment.

Each method offers different advantages and limitations. FNA is a simple and cost-effective method that provides valuable information. However, FNA specimens can be difficult to interpret in cases where there is a small sample, high sampling error, or a lack of a histological pattern (Hajdu et al., 1984). FNA sensitivity and accuracy are therefore positively affected by the presence of a cytopathologist on site during the biopsy procedure. Immediate assessment of the adequacy and quality of the sample also helps reduce the number of false negative results (Stewart et al., 1996; Nasuti et al., 2002; Wallace et al., 2004). CNB is more sensitive when defining benign lung lesions. Moreover, it is more accurate at characterizing lymphoproliferative diseases and subtyping malignancies, where the assessment of

${ }^{1}$ Department of Pathology, Sina Hospital, Tehran University of Medical Sciences, ${ }^{3}$ Department of Pathology Hazrat Rasool-e-Akram Hospital, ${ }^{7}$ Department of Pathology, Oncopathology Research Center, Iran University of Medical Sciences, Tehran, ${ }^{2}$ Department of Pathology, Shiraz University of Medical Sciences, Shiraz, Iran, ${ }^{4}$ A.T. Still University, Arizona, USA, ${ }^{5}$ Keiser Permanente Fontana California USA ${ }^{6}$ Department of Radiation Oncology, Ludwig-Maximilians University, München, Germany *For correspondence: sadeghipour.ar@iums.ac.ir, sadeghipour.alireza@gmail.com 
tissue architecture is crucial to the diagnosis (Sagel et al., 1978; Kim et al., 2002). However, CNB is more invasive and although considered to be generally safe, it may be associated with increased complications (Norenberg et al., 1974; Arnold et al., 2002).

There are conflicting data in the literature regarding the accuracy and usefulness of these techniques for the evaluation of intrathoracic lesions (Bocking et al., 1995; Arakawa et al., 1996; Greif et al., 1999; Nasuti et al., 2002; Kravtsov et al., 2014). Most previous reports present a limited number of cases and few studies have directly compared these two methods when treating the same patient (Gong et al., 2006). The purpose of this study was to compare the diagnostic accuracy of concurrent FNA and $\mathrm{CNB}$ of intrathoracic lesions in order to obtain reliable data on the advantages and disadvantages of each technique.

\section{Materials and Methods}

From October 2008 to September 2010, 127 patients at our center underwent sequential percutaneous computed tomography (CT)-guided FNA and CNB procedures for evaluation of intrathoracic lung lesions. The study was conducted with the approval of institutional review committees. Clinical and radiological information for these patients was made available for review. 98 out of 105 lesions were pulmonary lesions, five lesions involved thoracic wall and pulmonary parenchyma and the remaining two lesions were mediastinal.

Transthoracic CT-guided FNA was performed using a 20- or 22-gauge aspirating needle through a coaxial 18-gauge guide needle (Cook, Bloomington, IN). Two or three FNA passes were usually made. Direct smears were air-dried for Wright staining or fixed in $95 \%$ ethanol for Papanicolaou staining. An on-site pathologist was available for immediate assessment of specimen adequacy and triage the specimen for additional studies, including to send fresh sample to microbiology laboratory and tuberculosis referral center for culture, staining and if indicated PCR studies. In our study, an aspirate sample was considered to be inadequate if it was unrepresentative (i.e., markedly hypocellular, contained only blood, normal lung parenchyma and histiocytes). The subsequent cytological diagnosis was based on the cytomorphological features of the smears and special stains for microorganisms such as acid-fast stain for mycobacteria, nocardia and actinomycetes, Methanamine silver and PAS for fungi and Gram stain for Bacteria. Stainings were performed on previously prepared unstained smears if available.

CNB was performed in all cases using a 20-gauge, coaxial, automated cutting needle biopsy system (Cook). Two to four cores were usually obtained. The core biopsy specimen was transferred to $10 \%$ buffered formalin fixative. The formalin-fixed core tissues were embedded in paraffin, sectioned, and stained with H\&E. Immunohistochemical stains were applied on all CNB samples from cases with malignant lesions using a panel including CK7, CK20, p63, TTF-1 and CDX2 in order to define histologic types and origin of the tumoral cells (i.e. primary tumor versus metastatic ). Additional markers (such as synaptophysin, chromogranin A,
CD99, CD45 and etc.) were used in next steps based on histomorphological and immunohistochemical findings. Special stains were performed when indicated.

Two pathologists, who were not aware of the reported results in advance, assessed FNA and CNB samples independently. In other words, for blind randomization purposes, they were not able to extrapolate the results of FNA samples toward CNB samples or vice versa. The major diagnostic categories for FNA and CNB were malignant, indeterminate or atypical (when cytologic smears had atypical cells which didn't full-fill the criteria of malignancy or histologic sections mainly formed by necrotic tissue containing few atypical cells in between), negative for malignancy (NFM)-benign specific lesions, NFM- non-specific lesion (specimens with non-specific inflammation and/or fibrosis), inadequate. For cases classified as positive for malignancy, an attempt was made to determine the histological type, to understand whether it was a primary or metastatic lesion, and, if it was a metastasis, to specify its origins.

In cases of benign lesions, if the pathological findings were compatible with the radiological and clinical impressions, then these were defined as benign-specific lesions; this group included lesions such as granulomatous lesions, bronchiolitis obliterans organizing pneumonia, organizing bacterial pneumonia and hydatid cysts. If biopsy material comprised only normal parenchyma, fibrosis or non-specific inflammation, then it was classified as a benign non-specific lesion.

The diagnostic accuracy of each modality and the combination of both was assessed by comparing the initial diagnosis with the final diagnosis of the lesion. The final diagnosis was made by a combination of information from resected specimen revisions (in five cases) and/or definitive biopsy diagnosis (FNA and CNB) with consideration of immunohistochemical results, clinical and radiological data, microbiologic studies, follow up data and response to treatment modalities. All cases with final diagnoses of malignancy had unequivocal pathological findings. For those cases with diagnosis of " benign-nonspecific lesion" a follow-up studies were carried out for at least one year in order to rule out undiagnosed malignancy or specific benign lung lesions or masses.

\section{Results}

Of 127 cases included in the study 22 cases were excluded from further analysis because of lack of enough histologic and cytological material and/or radiologic or clinical evidence to determine the nature of the lesions. Of 105 cases that constituted the final cohort $63(60 \%)$ were male and $42(40 \%)$ were female. Median age for all 105 cases was 65 years (63 years for females and 65.5 years for males).The occurrence of lung lesions was higher in left lower lobe and right upper lobe. Location of mass did not affect the FNA or CNB results. Malignant tumors were diagnosed in $73(69.5 \%)$ and benign lesions in 32 (30.5\%) cases. Among these 105 cases, FNA and CNB findings were in complete agreement in 63 cases $(60 \%), 39$ of which were malignant and 24 were benign. Among the 73 malignant cases, 59 were primary epithelial tumors (five 

Table 1. Diagnostic Accuracy of FNA, CNB and Both with Application of IHC on CNB Samples in 73 Malignant intra-thoracic Lesions

\begin{tabular}{|c|c|c|c|}
\hline Final diagnosis & FNA & CNB & $\begin{array}{l}\mathrm{FNA}+\mathrm{CNB} \\
\mathrm{h} \text { application of } \\
\mathrm{HC} \text { on } \mathrm{CNB})\end{array}$ \\
\hline \multicolumn{4}{|l|}{ Epithelial tumors } \\
\hline Adenocarcinoma $(n=35)$ & $12(34.3)$ & $28(80)$ & $35(100)$ \\
\hline Primary $(n=27)$ & -- & --- & $27(100)$ \\
\hline Metastatic $(n=8)$ & --- & --- & $8(100)$ \\
\hline Squamous cell ca. $(n=24) *$ & $13(54.2)$ & $20(83.3)$ & $20(83.3)$ \\
\hline Typical carcinoid $(n=1)$ & $0(0)$ & $1(100)$ & $1(100)$ \\
\hline Atypical Carcinoid $(n=2) \ddagger$ & $0(0)$ & $0(0)$ & $2(100)$ \\
\hline Poorly Differentiated neuroendocrine carcinoma, small cell (small cell ca.) $(n=5)$ & $5(100)$ & $5(100)$ & $5(100)$ \\
\hline \multicolumn{4}{|l|}{ Non-Epithelial tumors } \\
\hline Sarcoma $(n=2)$ & $0(0)$ & $2(100)$ & $2(100)$ \\
\hline Hodgkin Lymphoma $(n=2)$ & $1(50)$ & $2(100)$ & $2(100)$ \\
\hline Primitive Neuroectodermal tumor (Small round malignant tumor) $(n=2) \dagger$ & $2(100)$ & $1(50)$ & $2(100)$ \\
\hline
\end{tabular}

- FNA, Fine-needle Aspiration; CNB, Core needle Biopsy; IHC, Immunohistochemical staining; -Data are given as number (percentage); *Four cases show mainly necrotic material or few atypical cells in FNA and CNB samples and final diagnosis was made on resection specimens; $†$ Diagnosed as "Small Round Malignant Tumor" by FNA \& CNB alone; $\$$ Erroneously diagnosed as "Squamous cell carcinoma" by CNB and "Non- small Cell Carcinoma" by FNA; ††Diagnosis of "Non-small cell Carcinoma" which made by FNA and CNB samples alone are not included

Table 2. Diagnostic Accuracy of FNA and CNB in 105 intra-thoracic Lesions*

\begin{tabular}{|c|c|c|c|c|}
\hline & \multicolumn{4}{|c|}{ Diagnosis } \\
\hline & $\begin{array}{l}\text { Benign } \\
\text { Specific } \\
\text { Lesion }\end{array}$ & Malignant & $\begin{array}{c}\text { Indeterminate } \\
\text { Atypical } \dagger\end{array}$ & $\begin{array}{l}\text { e/ NFM } \\
\text { (non-specific } \\
\text { lesion) } \$\end{array}$ \\
\hline FNA & $1(6.3)$ & $63(86.3)$ & $10(13.7)$ & $31(61.3)$ \\
\hline $\mathrm{CNB}$ & $13(81.3)$ & $68(93.2)$ & $4(5.5)$ & $19(95)$ \\
\hline $\mathrm{FNA}+\mathrm{CNB}$ & $13(81.3)$ & $69(94.5)$ & $4(5.5)$ & $19(100)$ \\
\hline
\end{tabular}

-FNA: Fine needle aspiration CNB: Core Needle Biopsy NFM: Negative for Malignancy; -Based on final diagnosis total number of malignant cases was 73 and benign lesions with a specific diagnosis were 16 ; *Data are given in "Number of cases (accuracy)"; †Cytological smears with atypical cells which didn't full-fill the criteria of malignancy or histologic sections with mainly necrotic tissue containing few atypical cells in between; $\ddagger$ Specimens with non-specific inflammation and/or fibrosis

Table 3. Diagnostic Accuracy of FNA, CNB and Both in Assessment of 32 Benign Lesions

\begin{tabular}{lrrr}
\hline Final Diagnosis & FNA & CNB & FNA+CNB \\
\hline NFM-Benign Specific Lesion $(\mathrm{n}=16)$ & & \\
Granulomatous inflammation $(\mathrm{n}=5)^{*}$ & $1(20)$ & $5(100)$ & $5(100)$ \\
Organizing bacterial pneumonia $(\mathrm{n}=8)$ & $0(0)$ & $5(62.5)$ & $5(62.5)$ \\
BOOP $(\mathrm{n}=1)$ & $0(0)$ & $1(100)$ & $1(100)$ \\
Hydatid cyst $(\mathrm{n}=2)$ & $0(0)$ & $2(100)$ & $2(100)$ \\
Total $(\mathrm{n}=16)$ & $1(6.3)$ & $13(81.3)$ & $13(81.3)$ \\
\hline
\end{tabular}

NFM; Negative for malignancy, BOOP; Bronchiolitis obliterans organizing pneumonia; Data are given in number (percentage); *Including both caseating and non-caseating granulomatous lesions

were small cell "neuroendocrine" carcinomas, 27 were adenocarcinomas, 24 were SCC, one typical carcinoid and two atypical carcinoids), eight were metastatic carcinomas ( six from colon and two from breast)and six were nonepithelial (two Hodgkin lymphomas, two sarcomas and two primitive neuroectodermal tumors) (Table 1). The indeterminate/atypical diagnosis was made in $9.5 \%(\mathrm{n}=10)$ and $3.8 \%(n=4)$ of FNA and CNB cases, respectively.

The overall diagnostic accuracy rates of FNA, CNB and both are summarized in Table 2. The accuracy of
FNA and CNB for malignant tumors was $86.3 \%$ and $93.2 \%$, respectively. The $95 \%$ confidence interval for FNA was 79.25- 90.65 and for CNB was 87.33- 95.95. For epithelial malignant neoplasms, definitive diagnosis was made in 30 out of 67 (44.8\%) cases by FNA and 54 out of 67 (80.6\%) cases by CNB. Within this group, FNA and CNB had similar typing accuracy rates for small cell "neuroendocrine" carcinoma. CNB showed better results for all other types of epithelial neoplasms. But the atypical carcinoid cases were erroneously diagnosed as non-small cell carcinoma and squamous cell carcinoma in FNA and CNB, respectively. Interestingly, a single primary adenocarcinoma case was diagnosed in FNA only. All four indeterminate/atypical CNB cases underwent surgical resection which had squamous cell carcinoma in histopathologic examination. For non-epithelial malignant neoplasms, CNB also yielded better diagnostic accuracy than FNA ( $83.3 \%$ vs 50\%, respectively). It is notable that neither of both sarcoma cases was diagnosed by FNA. In addition, one out of two primitive neuroectodermal tumors (PNET) which was misclassified as small cell carcinoma by CNB before application of immunohistochemical panel. It is noteworthy that both PNET cases were classified as small round malignant tumor by FNA and combination of two methods increased the accuracy of diagnosis (Table 1) (Figure 1a-c).

16 out of 32 benign cases were proven to be benignspecific lesions. FNA was diagnostic in a single case with diagnosis of granulomatous inflammation and CNB in 13 cases, accounting for $3.1 \%$ and $40.6 \%$ of all benign cases, respectively. The diagnostic accuracy of FNA and CNB for benign specific lesions was $6.3 \%$ and $81.3 \%$, respectively. In this group, FNA and CNB showed discrepant results in 12 cases. Combination of both didn't increase the diagnostic accuracy for detection of these types of lesions. In three cases which initial diagnosis were benign non-specific lesion, resection of one and the results of bacteriologic studies in combination with clinical findings were in keeping with organizing bacterial 


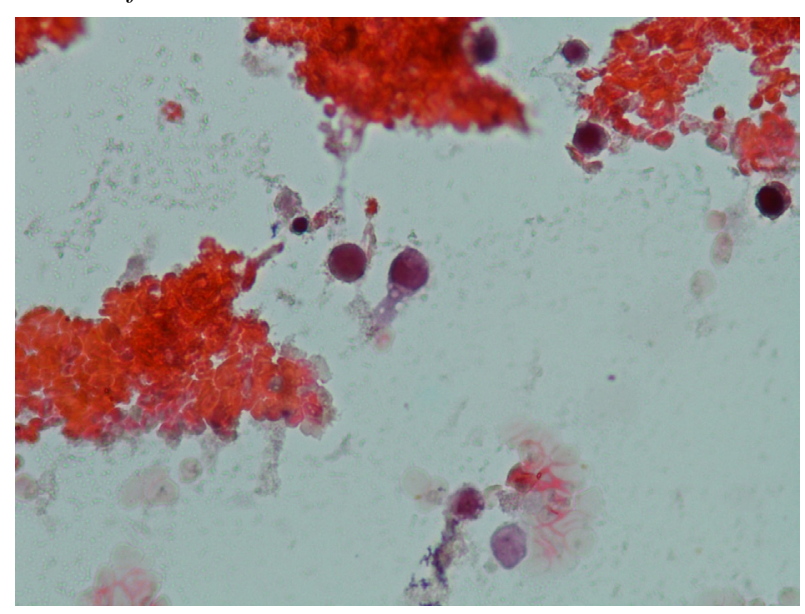

Figure 1. Fine-needle Aspiration Smears are Hypocellular Showing Isolated Small Round Tumoral Cells With High N/C Ratio, Dense Chromatin Pattern, Indistinct Nucleoli and Small amount of Pale Cytoplasm with Occasional Small Vacuoles. Background is dirty in favor of tumor diathesis, interpreted as "Small Round Malignant tumor". (Papanicolaou, X400)

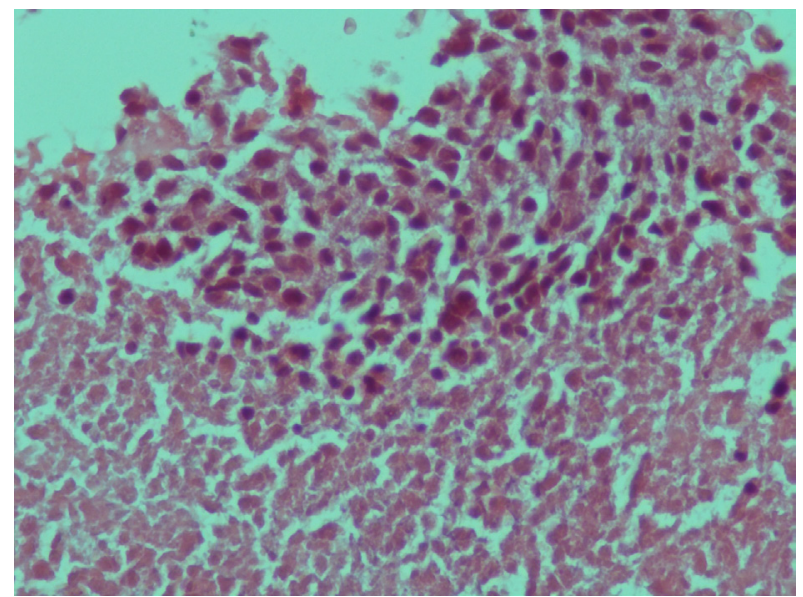

Figure 2. Concurrent Core Needle Biopsy Showing Sheet of Small Tumoral Cell in Associate with Large Areas of Necrosis Erroneously Interpreted as "Small Cell Carcinoma”.( H\&E, X400)

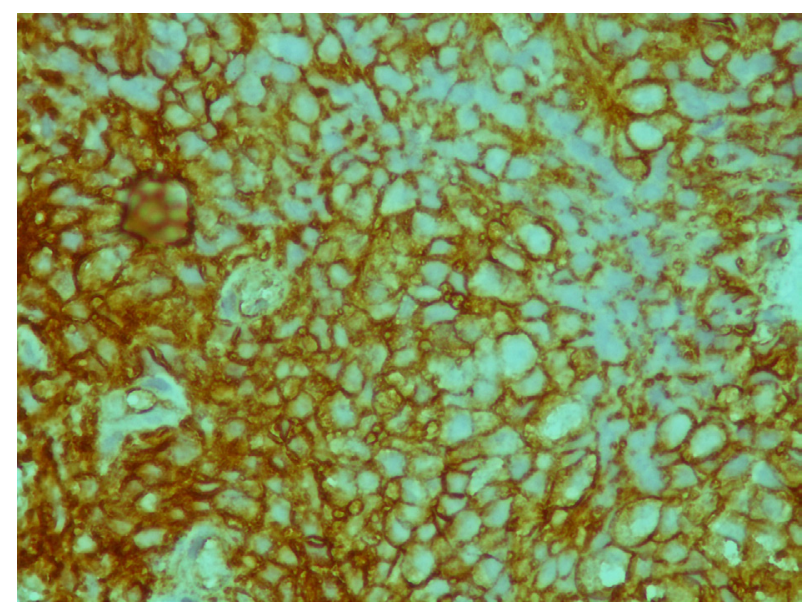

Figure 3. Immunohistochemical Staining of the Same Tumor (non necrotic area) Showing Strong Reactivity with CD99 Marker. The tumoral cells were negative for CK, Synaptophysin, Chromogranin, CD45 and myogenin. ( IHC pneumonia (Table 3 ). The remaining 16 benign cases showed benign-nonspecific features in both specimen types. Although there was no definitive tool to define the specific pathological nature of lesions, they were considered to be benign because all patients underwent benign clinical and radiological courses.

In this study, no false positive diagnosis of malignancy was observed with either technique. As previously reported, one false negative result was made in a case of primary adenocarcinoma with CNB technique.

\section{Discussion}

In 2008, lung cancer was the cause of $13 \%$ of all new cases of cancers and $18 \%$ of all cancer-related mortality, worldwide. The frequency of lung cancer in Iran is lower than in Europe and the USA (Jemal et al., 2011). On a nationwide scale, lung cancer ranks 7 th or 8 th in men and beyond 10th in women whereas on a global scale it ranks 1st and 4th in men and women, respectively (Emami et al., 2009; Mousavi et al., 2009). Despite lower frequency of lung cancer in Iran, the patients with this type of cancer have a short-term survival (Zahir and Mirtalebi, 2012) ranking 2nd in men and 3rd in women as the cause of cancer-related death (Mousavi et al., 2009). Tobacco smoking alone is the most important risk factor for lung cancer as it accounts for more than $70 \%$ of pulmonary carcinomas (WHO, 2013). According to recent studies, squamous cell carcinoma is the most common histopathological subtype of bronchogenic carcinoma in Iran and Turkey (Demirci et al., 2013; Hajmanoochehri et al., 2014)

Accurate pathological diagnosis is essential for selecting a suitable therapy for intrathoracic lesions. The skill of the operator, experience of the interpreter, and the nature of the lesion are among the factors that also affect diagnosis (Khouri et al., 1985; Spira et al., 2004; Kravtsov et al., 2014). FNA feasibility and availability justify the ready acceptance and wide use of this technique for lung masses. Previous studies have documented the reliability of FNA in the diagnosis of intrathoracic malignant tumors with reported accuracy rates of $80 \%$ to $95 \%$ (Moulton et al., 1993; Greif et al., 1999; Kim et al., 2002; Wallace et al., 2004; Kravtsov et al. 2014). However, controversy exists about the overall value, complications, and limitations of FNA and CNB in assessing lung masses. In a systematic review included eleven studies in total the reported range of diagnosis accuracy of FNA and CNB were $79.7 \%$ $91.8 \%$ and $89.0 \%-96.9 \%$ respectively. The range of accuracy for specific diagnosis of benign and malignant lesions were $40.4 \%-81.2 \%$ and $66.7 \%-93.2 \%$ by FNA and CNB respectively (Yao et al., 2012). Moreover, data that compare the use of both techniques performed in the same lesions are limited.

Our results demonstrate that for malignant tumors, the diagnostic accuracy of FNA $(86.3 \%)$ was acceptable but not generally comparable to that of CNB (93.2\%), while FNA showed lower diagnostic value in comparison to CNB in the histological typing of epithelial malignant neoplasms (44.8\% vs $80.6 \%$ ). Moreover, CNB showed better diagnostic accuracy than FNA in evaluation of 
non-epithelial malignant neoplasms (83.3\% vs $50 \%)$. Difficulty in aspirating mesenchymal neoplasms may result from entrapment of their cellular component in highly adhesive surrounding matrix, the fact that make CNB, an appropriate method for assessment of histologic architecture of the non-epithelial tumors and the performance of ancillary studies, which are essential for classifying of this type of neoplasms (Heilo et al., 1993; Pappa et al., 1996; Gong et al., 2006).

In previous studies, FNA and CNB have been shown to be complementary in the diagnosis of malignant tumors (Moulton et al., 1993; Kim et al., 2002). Despite the better diagnostic yields of CNB in our study, only a single case of malignant epithelial tumor was diagnosed only by FNA, whereas combining FNA and CNB slightly improved the diagnostic accuracy. These results suggest that the combination of FNA and CNB could diagnose most cases of malignancy and provide almost equivalent results as open biopsy, mediastinoscopy, and thoracoscopy without the associated high morbidity and cost.

The simplicity and cost-effectiveness of FNA could make it the primary procedure of choice for evaluating lung lesions, especially in patient with history of malignancy. In our study, although the tissue sampled by CNB allowed for the better architectural evaluation and classification of tumors, combination of FNA and CNB made better diagnostic yield in evaluation of small round malignant tumors. In addition, FNA material might be preferable to that acquired by CNB for microbiological studies. These cases manifest as Non-resolving pneumonias and therefore FNA can provide adequate material for appropriate microbiological and molecular studies, which are crucial in such cases.

Technical difficulties in aspirating representative material accounted for the majority of false negative results and indeterminate diagnoses made on FNA samples. Poor technique of smear preparation and fixation are other contributory factors. Immediate assessment by an on-site pathologist or cytopathologist improves the overall diagnostic yield by providing information about adequacy of FNA sample and if it is representative of the target lesion or not. In addition, it provide a background for better assessment of the sample by allowing triage of cases for appropriate workup (Conces et al., 1987; Austin et al., 1993; Wallace et al., 2004).

Although FNA offers the reliable diagnosis of malignant lesions, its ability to identify a benign lesion is questionable. Specific diagnoses of benign lesions have varied from $12 \%$ to $50 \%$ in previous studies (Moulton et al., 1993; Greif et al., 1999; Gong et al., 2006). According to some reports, core biopsy is superior to FNAB in diagnosis of benign thoracic lesions, mediastinal tumors, determination of cancer cell-type and predicting cancernegative findings (Beslic et al., 2012). In the present study, diagnostic accuracy of FNA for benign specific lesions was very low (6.3\%), while CNB improved the accuracy of a specific diagnosis for benign cases by $81.3 \%$. In our study, FNA and CNB showed discrepant results in 12 cases. In addition, combination of both techniques didn't increase the diagnostic accuracy for detection of these types of lesions. Indeed the difference between the results of these two procedures stands on the difference in capabilities in assessment of benign lesions. Unlike the diagnosis of malignant tumors for which individual cell morphology provide significant diagnostic information, diagnosis of benign specific lesions often depends on their histologic architecture, which frequently lost or destructed in FNA specimen.

Clinically and radiologically, benign lesions with benign cytologic and/or core biopsy findings might require follow-up only without surgical intervention, whereas a lesion with benign findings on FNA or CNB but clinical and/or radiologic evidence suggesting malignancy warrants re-biopsy or surgical excision (Gong et al., 2006). In our study, the interpretation of "NFM - nonspecific lesion" was reserved for those cases that were supported fully by negative clinical, radiologic and other findings. Four lesions were initially classified as atypical/ indeterminate by both methods but had clinical and radiologic features suspicious of malignancy were followed by surgical resection; all confirmed to have squamous cell carcinoma after histopathologic examination.

As mentioned earlier, FNA samples may have insufficient cells to interpret, but CNB mostly provided good tissue, which may not be sufficient as a pneumonectomy specimen. On these occasions, immunohistochemical methods could play an important role in the diagnostic evaluation of biopsy samples (Montezuma et al., 2013). For example, squamous cell carcinomas may form glandlike structures similar to adenocarcinomas, which are easily diagnosed by using a small immunohistochemical panel. In our study, few cases had surgical tissue specimens available and most final diagnoses were made with the immunohistochemical work up of CNB samples.

CT-guided transthoracic needle biopsy is generally regarded as a safe procedure with limited morbidity and extremely rare mortality. Complications of transthoracic needle biopsy include pneumothorax, hemoptysis, hemothorax, infection, and air embolism, with the most common complication as pneumothorax (Boskovic et al., 2014). Risk factors for the development of biopsy-related pneumothorax include the presence of chronic obstructive pulmonary disease (COPD), small lesion size, a long needle path, repeated pleural puncture, and the absence of a history of ipsilateral surgery (Dennie et al., 2001)

In conclusion, accurate pathological diagnosis is imperative for determining an optimal therapy for intrathoracic lesions. FNA and CNB offer different advantages and limitations for diagnosing lung lesions. Our findings suggest that FNA is comparable to CNB in the diagnosis of malignant epithelial lesions whereas diagnostic accuracy of CNB for nonepithlial malignant neoplasms is superior to FNA. Combining FNA and CNB methods slightly improves the diagnostic accuracy for malignant lesions. We suppose that Combination of FNA and CNB should be the preferred approach when benign lesions are suspected. Further, for the histological typing of tumors and for examining tumor origin, an immunohistochemical work up plays an important role. 


\section{References}

Arakawa H, Nakajima Y, Kurihara Y, Niimi H, Ishikawa T (1996). CT-guided transthoracic needle biopsy: a comparison between automated biopsy gun and fine needle aspiration. Clin Radiol, 51, 503-6.

Arnold B W, Zwiebel W J (2002). Percutaneous transthoracic needle biopsy complicated by air embolism. AJR Am J Roentgenol, 178, 1400-2.

Austin JH, Cohen MB (1993). Value of having a cytopathologist present during percutaneous fine-needle aspiration biopsy of lung: report of 55 cancer patients and meta-analysis of the literature. AJR Am J Roentgenol, 160, 175-7.

Beslic S, Zukic F, Milisic S (2012). Percutaneous transthoracic $\mathrm{CT}$ guided biopsies of lung lesions; fine needle aspiration biopsy versus core biopsy. Radiol Oncol, 46, 19-22

Bocking A, Klose KC, Kyll HJ, Hauptmann S (1995). Cytologic versus histologic evaluation of needle biopsy of the lung, hilum and mediastinum: sensitivity, specificity and typing accuracy. Acta Cytol, 39, 463-71.

Boskovic T, Stanic J, Pena-Karan S, et al (2014). Pneumothorax after transthoracic needle biopsy of lung lesions under CT guidance. J Thorac Dis, 6, 99-107

Conces DJ Jr, Schwenk GR Jr, Doering PR, et al (1987). Thoracic needle biopsy: improved results utilizing a team approach. Chest, 91, 813-6.

Demirci E, Daloglu F, Gundogdu C, et al (2013). Incidence and clinicopathologic features of primary lung cancer: a NorthEastern Anatolia region study in Turkey (2006-2012). Asian Pac J Cancer Prev, 14, 1989-93.

Dennie CJ, Matzinger FR, Mariner JR, et al (2001). Transthoracic needle biopsy of the lung: results of early discharge in 506 outpatients. Radiology, 219, 247-51

Emami Razavi SH, Aaghajani H, Haghazali M, et al (2009). The most common cancers in Iranian women. Iranian J Publ Health, 38, 109-12.

Gong Y, Sneige N, Guo M, Hicks ME, Moran CA (2006). Transthoracic fine-needle aspiration vs concurrent core needle biopsy in diagnosis of intrathoracic lesions: a retrospective comparison of diagnostic accuracy. Am J Clin Pathol, 125, 438-44.

Greif J, Marmor S, Schwarz Y, Staroselsky AN (1999). Percutaneous core needle biopsy vs fine needle aspiration in diagnosing benign lung lesions. Acta Cytol, 43, 756-60.

Greif J, Marmur S, Schwarz Y, Man A, Staroselsky AN (1998). Percutaneous core cutting needle biopsy compared with fine-needle aspiration in the diagnosis of peripheral lung malignant lesions: results in 156 patients. Cancer, 84, 144-7.

Hajdu SI, Melamed MR (1984). Limitations of aspiration cytology in the diagnosis of primary neoplasms. Acta Cytol, 28, 337-45.

Hajmanoochehri F, Mohammadi N, Zohal MA, et al (2014). Epidemiological and Clinicopathological Characteristics of Lung Cancer in a Teaching Hospital in Iran. Asian Pac J Cancer Prev, 15 (6), 2495-2500

Heilo A (1993). Tumors in the mediastinum: US-guided histologic core-needle biopsy. Radiology, 189, 143-6.

Jemal A, Bray F, Center M, et al (2011). Global cancer statistics. CA Cancer J Clin, 61, 69-90.

Khouri NF, Stitik FP, Erozan YS, et al (1985). Transthoracic needle aspiration biopsy of benign and malignant lung lesions. AJR Am J Roentgenol, 144, 281-8.

Kim HK, Shin BK, Cho SJ, et al (2002). Transthoracic fine needle aspiration and core biopsy of pulmonary lesions. A study of 296 patients. Acta Cytol, 46, 1061-8.

Kravtsov V, Sukmanov I, Yaffe D, et al (2014). Diagnostic aspects of fine needle aspiration for lung lesions: series of
245 cases. Asian Pac J Cancer Prev, 15, 9865-9

Montezuma D, Azevedo R, Lopes P, et al (2013). A panel of four immunohistochemical markers (CK7, CK20, TTF-1, and p63) allows accurate diagnosis of primary and metastatic lung carcinoma on biopsy specimens. Virchows Arch, 463, 749-54.

Moulton JS, Moore PT (1993). Coaxial percutaneous biopsy technique with automated biopsy devices: value in improving accuracy and negative predictive value. Radiology, 186, 515-22.

Mousavi SM, Gouya MM, Ramazani R, et al (2009). Cancer incidence and mortality in Iran. Ann Oncol, 20, 556-63.

Nasuti JF, Gupta PK, Zubair WB (2002).Diagnostic value and cost-effectiveness of on site evaluation of fine-needle aspiration specimens: review of 5.688 cases. Diagn Cytopathol, 27, 1-4.

Norenberg R, Claxton CP Jr, Takaro T (1974). Percutaneous needle biopsy of the lung: report of two fatal complications. Chest, 66, 216-8.

Pappa VI, Hussain HK, Reznek RH, et al (1996). Role of image guided core-needle biopsy in the management of patients with lymphoma. J Clin Oncol, 14, 2427-30.

Sagel SS, Ferguson TB, Forrest JV, et al (1978). Percutaneous transthoracic aspiration needle biopsy. Ann Thorac Surg, 26, 399-405.

Spira A, Ettinger DS (2004). Multidisciplinary management of lung cancer. $N$ Engl J Med, 50, 379-392.

Stewart CJ, Stewart IS (1996). Immediate assessment of fine needle aspiration cytology of lung. J Clin Pathol, 49, 839843.

World Health Organization (2013). Cancer. Factsheet.

Wallace AB, Suh RD (2004). Percutaneous transthoracic needle biopsy: special considerations and techniques used in lung transplant recipients. Semin Intervent Radiol, 21, 247-58.

Yao X, Gomes MM, Tsao MS, et al (2012). Fine-needle aspiration biopsy versus core-needle biopsy in diagnosing lung cancer: A systematic review. Current oncology, 19, 16-27

Zahir ST, Mirtalebi M (2012).Survival of patients with lung cancer, Yazd, Iran. Asian Pac J Cancer Prev, 13, 4387-91. 